\title{
Socialisation familiale, éducation scolaire et formation en entreprise
}

Comparaison France-Brésil-Japon

Helena Sumiko Hirata

\section{OpenEdition}

\section{Journals}

Édition électronique

URL : http://journals.openedition.org/ries/4302

DOI : $10.4000 /$ ries.4302

ISSN : 2261-4265

\section{Éditeur}

Centre international d'études pédagogiques

Édition imprimée

Date de publication : 1 mars 1994

Pagination : 47-56

ISSN : 1254-4590

\section{Référence électronique}

Helena Sumiko Hirata, «Socialisation familiale, éducation scolaire et formation en entreprise », Revue internationale d'éducation de Sèvres [En ligne], 01 | 1994, mis en ligne le 20 avril 2015, consulté le 01 mai 2019. URL : http://journals.openedition.org/ries/4302 ; DOI : 10.4000/ries.4302

Ce document a été généré automatiquement le 1 mai 2019.

(c) Tous droits réservés 


\title{
Socialisation familiale, éducation scolaire et formation en entreprise
}

\author{
Comparaison France-Brésil-Japon
}

Helena Sumiko Hirata

\section{NOTE DE L'AUTEUR}

Ce texte reprend quelques résultats de recherches empiriques réalisées au Brésil, en France et au Japon depuis 1980 sur les aspects techniques et socioculturelles de l'organisation du travail dans des entreprises multinationales, résultats concernant l'éducation et la formation professionnelle. Nous devons nombre de données comparatives sur le système éducatif et de formation professionnelle au Brésil et en France à Jean-Jacques Paul, économiste de l'éducation (à l'IREDU et à l'université de Lyon), avec qui nous avons effectué une recherche comparative France-Brésil en collaboration. Une première version de ce texte a été publiée en portugais dans Educaçao $e$ Sociedade, n 31 , Revista quadrimestral de Ciencias da Educaçao, décembre 1988, Cortez Editora, Sao Paulo.

1 L'intérêt des spécialistes du travail et de l'entreprise pour la formation professionnelle n'a cessé de croître dans les pays occidentaux dans les années 80. En effet, les mutations technologiques rapides et l'émergence de nouveaux modèles d'organisation industrielle fondés sur l'emploi d'une main-d'œuvre plus qualifiée et mieux formée, auparavant moins demandée dans le cadre de la production de masse fordiste, ont suscité un débat sur les nouvelles exigences en matière d'éducation et de formation. Ce sujet est devenu un lieu de confrontation entre spécialistes du travail et de l'éducation (L. Tanguy, 1986).

2 Parmi les «modèles » internationaux, l'exemple japonais a été, et continue à être, un de ceux qui ont été le plus souvent évoqués, sans doute en raison de la grande diffusion dans l'entreprise japonaise des innovations technologiques et organisationnelles, mais aussi en raison de son succès économique. Nous l'examinons ici à la lumière des cas du Brésil et de la France. 
3 Les caractéristiques quasi uniques de la formation du travailleur japonais par rapport à celle de ses homologues occidentaux, tant des pays en voie de développement que des pays très industrialisés, sont bien mises en évidence par la comparaison internationale. Nous analysons ici comment ces caractéristiques ont leur origine non seulement dans l'organisation du travail industriel et dans le système d'emploi (M. Aoki, 1991), mais aussi dans le modèle éducatif au sens large comprenant la socialisation et les relations familiales en vigueur dans la société japonaise. Partant d'une critique du déterminisme technologique appuyée sur la comparaison internationale du travail industriel, nous arrivons à la conclusion que les différences liées à l'organisation du travail dans des entreprises de pays différents dépendent plus de singularités d'ordre sociétal, et parmi elles l'éducation scolaire et le type de socialisation au sein de la famille. Nous exposerons successivement les caractéristiques de la socialisation familiale, de l'éducation scolaire générale, de la formation professionnelle externe puis interne à l'entreprise.

\section{La socialisation familiale}

Même si la mère éducatrice est une catégorie universelle (Rolf Nemitz, 1986), puisque toute une série d'apprentissages se font à l'intérieur de la sphère familiale, son intervention connaît selon les pays des modulations différentes. Ainsi, la responsabilité primordiale - pratiquement exclusive - des femmes japonaises dans l'éducation des enfants semble être un corollaire de leur position sur le marché du travail (exclusion du marché du travail, surtout industriel, à partir du mariage; emploi précaire à temps partiel après l'éducation des enfants) et de la prééminence absolue du monde professionnel pour les hommes. En France, l'éducation des enfants s'accomplit de façon plus significative à l'extérieur : les équipements collectifs y sont plus nombreux qu'au Japon (crèches à partir de l'âge de deux mois, garderies, etc.), le travail professionnel de la femme mariée est le modèle dominant (les statistiques françaises montrent que, dès les années 80 , le passage à l'inactivité se fait seulement après la naissance du troisième enfant). Au Brésil, il semble y avoir une différenciation plus accentuée des modèles selon les classes sociales, étant donné le recours possible dans ce pays au travail domestique rémunéré (employées de maison, femmes de ménage, gardes d'enfant), mais la mère se voit encore attribuer socialement une plus grande responsabilité qu'en France dans l'éducation des enfants et sa présence sur le marché du travail est plus faible.

\section{L'éducation scolaire générale}

5 La France se situe entre deux cas extrêmes : celui que représente le Japon avec un haut niveau de scolarisation, où plus de $99 \%$ de la population possède un diplôme du premier degré et plus de $90 \%$ de ceux-ci rentrent dans l'enseignement de second degré (Statistics Bureau, 1987 : 130), et celui du Brésil avec un taux élevé d'évasion scolaire, de 16 à $40 \%$ sur les huit années du premier degré, et $25 \%$ de la population d'âge scolaire coupés du système éducatif (Brésil, données pour l'État de São Paulo) ${ }^{1}$. Au Japon, ce taux d'évasion est inférieur à $1 \%$ au cours des neuf ans de scolarité obligatoire (Nohara, 1983, p. 3).

Plus intéressante est la comparaison entre les différentes modalités de relation entre école et entreprise : leurs liens sont très ténus au Brésil et en France par rapport au Japon, où le recrutement est fait chaque année par les grandes entreprises, à la fin de 
l'année scolaire (mars), dans les écoles secondaires et les universités. Cette fonction de pourvoyeuse d'emplois de l'école est institutionnalisée, coordonnée par le ministère du travail (Nohara, 1983, p. 26), même s'il y a négociation directe entre les chefs du personnel des grandes entreprises et la direction des écoles. Le rapport entre l'école et l'entreprise existe aussi au niveau de la sélection: les écoles les plus recherchées fournissent la main-d'œuvre pour les entreprises les plus connues, les écoles de prestige moindre fournissent les entreprises moins valorisées, et ainsi de suite.

\section{L'enseignement technique et la formation professionnelle hors de l'entreprise}

7 La prééminence de la formation technique scolaire en France, confirmée par sa reconnaissance dans les conventions collectives, contraste avec son développement relativement moindre au Brésil et au Japon.

$8 \mathrm{Au}$ Brésil, nous rencontrons trois types de formation hors de l'entreprise : les écoles techniques, partie intégrante du système officiel, bien que certaines d'entre elles soient privées; l'enseignement professionnel non-officiel, qui offre des cycles de formation moyens et courts, sans contrôle pédagogique, pour des professionnels d'un niveau relativement bas; et les SENAI-SENAC, (Services nationaux d'apprentissage industriel et commercial) financés par une contribution obligatoire des entreprises ( $1 \%$ de la masse salariale) et sous contrôle du ministère du travail. Il faut noter que cette contribution de l'entreprise à la formation professionnelle existe aussi en France, mais pas au Japon (Nohara, 1983, p. 7, note 1). Le SENAI forme dans ses centres la main-d'œuvre qualifiée et semi-qualifiée liée à la production; dans les entreprises, il envoie ses apprentis ou dispense des cours ponctuels.

9 En France, d'après l'Enquête sur l'emploi du ministère du travail de 1990, environ un quart des 23 millions de personnes qui constituent la population active ${ }^{2}$ possède un diplôme professionnel équivalent au niveau secondaire. Il existe une grande variété d'écoles techniques qui assurent des formations pour les postes de travail les plus divers, qui font partie du système officiel, et qui délivrent un nombre élevé de diplômes nationaux. Le nombre de diplômes professionnels pour la France paraît élevé, mais dans la réalité il est faible comparé à celui de l'Allemagne; une étude comparative a montré que sur un échantillon d'ouvriers de production des deux pays, $40 \%$ des ouvriers français étaient en possession d'un diplôme professionnel, contre $75 \%$ des ouvriers allemands (M. Maurice, F. Sellier, J.-J. Sylvestre, 1982).

10 Au Japon, comme au Brésil, coexistent des écoles techniques en majorité publiques et appartenant à l'appareil éducatif officiel, des écoles spéciales d'enseignement professionnel de niveau relativement bas, et des centres publics d'apprentissage professionnel. Les lycées techniques et les collèges techniques supérieurs font partie du système officiel et sont notablement plus nombreux qu'au Brésil. Les études y durent cinq ans et sont équivalentes au niveau du second degré plus une partie d'enseignement supérieur. Ils forment des techniciens dans plus de 200 spécialités industrielles dont seulement $2 \%$ d'étudiantes (Nohara, 1983, p. 11). L'enseignement professionnel nonofficiel présente des caractéristiques semblables à celles du même secteur au Brésil, bien qu'il soit contrôlé depuis peu par le ministère de l'Éducation nationale (Nohara, 1983, p. 14). Il offre un enseignement court (6-24 mois) hors du système scolaire, débouchant sur des secteurs tels que les professions de santé, de secrétariat et de la comptabilité. Près 
de $72 \%$ des élèves sont des femmes, ce qui indique $a$ contrario, le bas niveau technique de ce type d'enseignement. Enfin, les centres nationaux et départementaux d'apprentissage professionnel, contrôlés par le ministère du travail, dispensent une formation de base de deux types ( 3200 heures de cours sur deux années pour ceux qui sortent du premier degré, et un cycle avancé de un ou deux ans pour ceux qui sortent du second degré) avec trois-quarts des heures d'enseignement consacrées aux travaux pratiques. Les deux types de formation préparent à l'exercice d'une centaine de professions (charpentier, mécanicien, plombier, soudeur, électricien, dessinateur industriel, etc.), mais les diplômés semblent s'orienter massivement vers le secteur artisanal et non vers l'industrie, comme c'est le cas pour le SENAI.

11 Le fait que le système d'apprentissage technique débouche au Japon sur un diplôme national du ministère du travail (Ginô Kentei - certificat d'aptitude professionnelle) ne signifie pas que sa portée soit comparable à celle des diplômes français - nationaux et homogènes - et qui correspondent à des qualifications reconnues par les conventions collectives et matérialisées dans un système de classifications. Au Japon, de même qu'au Brésil, il n'y a pas de système homogène de diplômes professionnels socialement valorisés, ni de système de classifications. Dans la réalité, le rapport formation-emploi en France n'existe qu'en termes de correspondance précise entre des postes de travail et des niveaux de formation de l'éducation nationale. Les diplômes japonais tendent à être utilisés davantage dans le secteur artisanal que dans le secteur industriel proprement dit, et ils ne servent pas « comme régulateurs d'emplois sur le marché du travail » (Nohara, 1983, p. 13).

Bien que l'ensemble de l'appareil soit important, la formation professionnelle et technique dispensée par ces organismes n'est pas socialement valorisée, et leurs effectifs sont stables ou tendent à diminuer (Nohara, 1983). Ceci s'explique par le prestige de la formation de niveau universitaire autant que - et surtout, selon nous - par le poids de la formation à l'intérieur de l'entreprise, et par le prestige des " écoles d'entreprise » qui ont joué, historiquement et traditionnellement, un rôle stratégique dans la formation de main-d'œuvre qualifiée pour l'industrie (A. Gordon, 1983).

13 Ce qui est valorisé et reconnu au Japon et au Brésil, semble-t-il, c'est davantage l'expérience individuelle matérialisée par le temps de service que le diplôme: "La qualification ouvrière se forge, surtout, dans les grandes unités de production situées au centre des forces productives, par l'accumulation des expériences empiriques au sein même de la production. La qualification ouvrière ainsi constituée ne peut s'approprier une valeur sociale objectivable» (Nohara, 1983, p. 44; cf. aussi H. Hirata, 1987). D'où l'intérêt de considérer maintenant la formation ouvrière à l'intérieur de l'entreprise.

\section{La formation professionnelle à l'intérieur de l'entreprise}

14 La pratique habituelle des entreprises occidentales (françaises et brésiliennes) est de fournir peu de formation spécifique aux catégories ouvrières, en se limitant, en plus de la formation on the job, aux formations élémentaires en matière de sécurité ou de supervision (type TWI - Training Within Industry). Les catégories supérieures se voient offrir davantage d'occasions de formation, surtout sous forme de stages ou de cours en dehors de l'entreprise. Cette situation contraste fortement avec le cas japonais, où un 
nombre élevé d'heures de formation est donné aux ouvriers de production, complété par des «formations complémentaires qui suivent l'évolution de la carrière professionnelle " (Nohara, p. 14). Pour ne citer qu'un exemple, celui de la formation aux activités des cercles de contrôle de la qualité - petits groupes formés par les travailleurs d'une section de l'usine pour discuter régulièrement et résoudre par eux-mêmes les différents problèmes rencontrés sur leur lieu de travail (sur les CCQ, H. Hirata, 1984a) -, on peut relever que le nombre d'heures de formation attribué aux ouvriers de production est plus grand que celui attribué à la direction et aux cadres.

15 Le système de formation à l'intérieur de l'entreprise elle-même est extrêmement diversifié au Japon, et ouvre l'accès aux connaissances techniques délivrées par l'entreprise mais qui ont une équivalence avec les diplômes scolaires, puisque les « écoles d'entreprise » (au nombre de 305 selon Nohara, 1983) reçoivent l'agrément du ministère du travail.

Ces écoles remplissent diverses fonctions :

- formation technique de personnel qualifié, équivalente à une formation de niveau supérieur ;

- formation générale remplaçant l'instruction scolaire officielle, surtout de niveau secondaire ; ceci est fréquent dans les entreprises embauchant une main-d'œuvre féminine recrutée en milieu rural et qui, en échange du travail en usine textile ou électronique, reçoit une formation scolaire; cette fonction d'instruction générale des "écoles d'entreprise " était également assurée jusque dans les années 50 dans les grandes entreprises et dans les usines (sidérurgie, construction navale, par exemple), mais elle a disparu avec l'évolution du système éducatif officiel au Japon (Nohara, p. 14);

- formation des femmes aux «arts domestiques japonais»; cette formation semble être une forme de préparation «technique » aux capacités requises par le processus de travail dans les industries de main-d'œuvre féminine (cf. infra, résultats de la recherche);

- enfin, formation générale mais exhaustive sur l'entreprise, notamment son histoire, les comportements qu'elle attend de ses salariés, les objectifs de la direction.

17 Les formations apparaissent comme singulièrement différenciées selon le sexe. En raison de la jeunesse des ouvrières dans l'industrie, qui travaillent dans l'entreprise en général de 18 à 24 ans puis démissionnent pour se marier, la formation interne apparaît comme une extension de la socialisation familiale. Dans les dortoirs, sous l'autorité d'une directrice, unique femme d'âge mûr dans une entreprise industrielle japonaise, les ouvrières apprennent les règles de propreté et d'hygiène, les normes pour accomplir le travail domestique (faire les chambres, laver le linge), l'obéissance au «père-chef » de l'usine, et les « arts domestiques japonais » (art du bouquet - Ikebana-, cérémonie du thé, calligraphie, danse traditionnelle japonaise, couture du kimono, art culinaire). Cet apprentissage forme et perfectionne sans doute la « dextérité manuelle » tant recherchée pour les postes de travail féminins.

18 Toujours pour la même raison - la jeunesse du personnel- l'entreprise offre une extension de l'instruction scolaire. Les ouvrières du textile, par exemple, font du travail posté, et, en dehors de ce travail posté, elles suivent les études du cours secondaire supérieur, en général à l'intérieur de l'entreprise elle-même (le recrutement d'une partie des ouvrières de l'électronique et du textile se fait en effet au niveau du premier degré).

Après quatre ou cinq ans d'expérience professionnelle, les ouvrières japonaises ont un diplôme scolaire, la maîtrise des arts domestiques, et une « dot » constituée par l'épargne 
forcée (l'entreprise retient une partie du salaire mensuel pour les dépenses d'entretien et de logement, et une partie comme « économies pour l'avenir », H. Hirata : 1986, 1984b).

La formation technique proprement dite est limitée dans le cas des femmes, et la formation aux activités de CCQ est également sommaire, en rien comparable à celle fournie aux hommes. Les employeurs expliquent cette situation par la brièveté de la carrière féminine dans l'entreprise japonaise qui rend peu rentable un investissement plus approfondi dans la formation technique.

La formation professionnelle des hommes japonais à l'intérieur de l'entreprise est extrêmement diversifiée, et elle existe à tous les stades de la carrière professionnelle. Elle revêt des formes de recyclage plus ou moins originales, mais la plus importante à signaler est probablement celle acquise pendant plusieurs années d'expérience à l'intérieur d'un collectif de travail (nous faisons évidemment référence aux effectifs de notre échantillon, c'est-à-dire ouvriers de grandes entreprises qui font partie de l'ensemble des ouvriers stables, et non des ouvriers temporaires ou sous-payés). Cette formation, obtenue par des échanges informels et de tous les instants, par des pratiques de rotation de tâches et par la polyvalence, est sans aucun doute un trait distinctif de la formation en entreprise, qui contraste fortement avec le taux de rotation élevé des travailleurs dans l'entreprise brésilienne qui rend impossible cette modalité de formation (H. Hirata, 1984a) (même si, au Brésil, d'une entreprise à l'autre et d'un secteur à l'autre, il y a évidemment des différences sensibles dans les temps de service des effectifs ouvriers). Si le taux de turn over est moins élevé en France qu'au Brésil, il n'est pas nul pour autant, et le temps de service des ouvriers français est relativement moindre que celui des ouvriers japonais, bien que beaucoup plus important que celui des ouvriers brésiliens.

Une deuxième modalité de formation, particulière elle aussi aux entreprises japonaises, est celle qui s'obtient par la mobilité à l'intérieur du groupe industriel. Le perfectionnement professionnel s'obtient par la délocalisation du personnel d'un établissement à l'autre. Cette délocalisation obéit aussi à d'autres objectifs comme la promotion ou les besoins en main-d'œuvre expérimentée dans une nouvelle implantation industrielle (H. Hirata, 1986).

Cette mobilité dans l'entreprise existe aussi dans la grande entreprise brésilienne ou française, mais sa portée est beaucoup plus limitée puisqu'elle touche presque exclusivement les cadres (gérants et hauts responsables de l'entreprise). Au Japon, où elle est une forme de promotion et de recyclage, la mobilité touche une masse notablement plus importante de la main-d'œuvre, principalement à partir du premier échelon de la hiérarchie, mais aussi le personnel ouvrier.

24 Une troisième modalité, comparable aux systèmes de formation dans l'entreprise adoptés au Brésil ou en France et plus proche d'eux, a trait aux cours de formation: cours de formation de base ou spécifique au lieu de travail, pour les nouveaux recrutés, cours de formation complémentaire selon l'évolution de la carrière professionnelle et surtout au moment du passage à l'échelon hiérarchique supérieur.

Les " écoles d'entreprise » existent aussi au Brésil, cependant - et la différence nous paraît considérable -, leurs cours ne sont pas reconnus par le ministère du travail ou de l'éducation nationale, comme au Japon. Elles ne peuvent pas délivrer de diplômes équivalents aux cours externes, secondaires ou supérieurs. Le passage de contremaître à ingénieur grâce à une formation externe est déjà un cas rarissime, étant donné les critères de division sociale au Brésil (nous n'en avons rencontré qu'un seul exemple dans 
notre enquête). Au Japon, par exemple, dans un établissement d'électronique, quatre ingénieurs seulement sur quarante étaient diplômés d'une université; les trente-six autres avaient obtenu leur titre dans l'entreprise et y exerçaient leur fonction. éducation scolaire et formation professionnelle. Nous avons vu qu'il n'était pas possible d'analyser le système de formation à l'intérieur de l'entreprise sans le mettre en relation avec les très nombreuses sphères d'apprentissage qui existent dans la société, et par conséquent à l'extérieur de l'entreprise. L'étude comparative France-Allemagne du LEST (Laboratoire d'économie et sociologie du travail, CNRS), avait déjà montré les relations entre le système éducatif et les faits d'organisation industrielle (M. Maurice et al, 1982). Cependant, elle n'avait pas analysé la variable structure familiale qui est apparue dans notre recherche sous la forme d'une contribution essentielle de la famille dans l'activité professionnelle de l'ouvrier japonais. La productivité plus forte du travail dans l'entreprise, les pratiques particulières de formation professionnelle (comme la formation continue pour les $\mathrm{CCQ}$, ou la formation par la mobilité interne du salarié, sans sa famille) deviennent possibles grâce à la coopération de la famille et au sacrifice, de la part des enfants et de la femme, des heures de loisir et même de cohabitation.

Un second point à relever est la différence dans les modes de production et de reconnaissance de la qualification professionnelle entre des établissements comparables du point de vue de la technologie, de la taille et du produit. En France, l'existence d'un système de classification au niveau du secteur est associée à l'existence de diplômes nationaux. Leur absence et par conséquent celle d'un système de classification, fait qu'au Brésil l'ancienneté soit la variable la plus significative dans la reconnaissance de la qualification professionnelle pour les hommes, ce qui est assez proche des critères japonais : âge et ancienneté continuent à mesurer encore, de façon prédominante, la qualification dans la grande entreprise japonaise.

Quant au travail féminin, si les employeurs dans les établissements français, japonais et brésiliens reconnaissent les qualités propres à la main-d'œuvre féminine, ces qualités ne sont pas reconnues en tant que qualifications.

En dernier lieu, l'étude a monté l'importance des « qualifications tacites » (S. Wood, 1984), surtout en ce qui concerne la main-d'œuvre féminine et sa place respective dans les trois pays. Au Brésil et en France, les qualifications techniques, formelles, sont plus importantes que les qualifications tacites: la compétence, la performance, la capacité d'occuper un poste de travail, considérées comme des vertus fondamentales, indiquent l'importance de l'éducation officielle, de la formation professionnelle et de l'expérience professionnelle antérieure (la carrière) en rapport avec la socialisation familiale. L'importance des qualifications tacites a été constatée au Japon surtout, où des qualités comme le Ninjo et le $\mathrm{Jimbo}^{3}$ sont considérées comme essentielles dans le recrutement des chefs et des contremaîtres. Ces qualités personnelles se structurent dans la sphère de la sociabilité hors-entreprise, dans la socialisation familiale et scolaire, même si la personnalité du recruté est ensuite façonnée par l'entreprise. 
indications, nous retiendrons l'importance, dans l'étude des rapports entre travail et éducation, entre formation et emploi, d'appréhender ensemble les trois dimensions que représentent la famille, l'école et l'entreprise.

\section{BIBLIOGRAPHIE}

AOKI M. (1991), Économie japonaise, Paris, Economica.

GORDON A. (1983), Les rapports sociaux et le mouvement syndical dans l'Industrie lourde japonaise au XIX ${ }^{\mathrm{e}}$ siècle, Le mouvement social, janvier-mars.

HIRATA H (1984a), « Division internationale et taylorisme : Brésil, France et Japon », in Le Taylorisme, M. de Montmollin et O. Pastré (sous la direction de), Paris, éd. La Découverte.

(1984b) «Vie reproductive et production : famille et entreprise au Japon », In Le sexe du travail, ouvrage collectif, Grenoble, PUG. (Trad. portug. : O sexo do traballio. Sâo Paulo, Paz e Terra, 1987.)

(1986a) « Famille, travail et rapports hommes-femmes : réflexions à partir du cas japonais », in Actes des Ateliers de recherche, $\mathrm{n}^{\circ}$ 6, Université de Picardie, Amiens. (Trad. portug. : Revista Brasileira de Ciencias Socials, $\mathrm{n}^{\circ}$ 2, Sâo Paulo, ANPOCS).

HIRATA H. (éd.) (1992), Autour du « modèle » japonais, Paris, éd. L'Harmattan.

HIRATA H., HUMPHREY J. (1984), « Crise économique et division sexuelle du travail », Sociologie du Travail, 3/84, Paris, Dunod.

HUMPHREY J. (1987), Women and work in third world, London, Tavistock.

ISHIKAWA T. (1981), « Vocational training », Japanese Industrial Relations Series, Tokyo, the Japan Institute of Labour.

JONES B., WOOD S. (1984), «Qualifications tacites, division du travail et nouvelles technologies », in Sociologie du travail, numéro Nouvelles technologies dans l'industrie : l'enjeu des qualifications, 4/84, Paris, Dunod.

MAURICE M., SELLIER F. \& SYLVESTRE J.-J. (1982), Politique d'éducation et organisation industrielle en France et en Allemagne, Paris, PUF.

NEMITZ R. (1987), « La famille et l'école. Éléments d'une théorie marxiste de l'éducation », in Actuel Marx, $\mathrm{n}^{\circ} 1$, Paris, éd. L'Harmattan.

NOHARA H. (1983), Enseignement technique, qualification industrielle et marché du travail : le cas du Japon, Document LEST, 83-8, LEST-CNRS.

STATISTICS BUREAU (1987), Management and Coordination Agency, Statistical Handbook of Japan.

TANGUY L., (sous la dir. de) (1986), L'introuvable relation formation/emploi, Paris, La Documentation française. 


\section{NOTES}

1. Des données plus récentes sur l'évasion scolaire pour l'ensemble du Brésil montrent que ces indices demeurent assez élevés, avec de fortes inégalités régionales. Cf. M.J. Garcia Werebe, Grandezas e miserias do ensino no Brasil, $3^{\mathrm{e}}$ éd. actualisée, éd. Atlas, S. P., 1994 (sous presse).

Quant à la France, l'enquête sur l'emploi de 1990 montre que le tiers de la population active n'a pas le diplôme de fin du premier cycle et que seulement un quart de la population active a le niveau bac.

2. L'Enquête sur l'emploi de 1990 donne le chiffre de 6600000 sur les 24576000 qui constituent la population active.

3. Ninjo : sentiments humains, compassion, humanité ; Jimbo : estime, popularité, prestige.

\section{RÉSUMÉS}

Examen de l'exemple japonais à la lumière des cas du Brésil et de la France. L'analyse montre l'influence qu'exercent les singularités sociétales telles que la socialisation familiale et les formes d'enseignement sur les modalités d'organisation du travail.

\section{INDEX}

Index géographique : France, Brésil, Japon

Mots-clés : comportement social, éducation comparée, recherche comparative, relation écoleentreprise, système éducatif

\section{AUTEUR}

HELENA SUMIKO HIRATA

GEDISST-CNRS 